\title{
Influencia de la ganadería en los macroinvertebrados acuáticos en microcuencas de los Andes centrales de Colombia
}

\author{
Yuly Paulina Ramírez ${ }^{1}$, Lina Paola Giraldo ${ }^{1}$, María del Carmen Zúñiga ${ }^{1,2}$, Blanca Cecilia Ramos ${ }^{2}$ \\ \& Julián Chará ${ }^{1}$ \\ 1. Centro para la Investigación en Sistemas Sostenibles de Producción Agropecuaria -CIPAV, Carrera 25 No. 6-62, Cali, \\ Colombia; yulypaulinaramirez@gmail.com, lina@fun.cipav.org.co,julian@fun.cipav.org.co \\ 2. Grupo de Investigaciones Entomológicas, Departamento de Biología, Universidad del Valle, AA 25360. Cali, \\ Colombia; maczuniga@gmail.com, blancera@gmail.com
}

Recibido 16-VIII-2017. Corregido 27-IV-2018. Aceptado 06-VI-2018.

\begin{abstract}
Cattle raising influence over the aquatic macroinvertebrates in microbasins of central Andes, Colombia. In Colombia, livestock activity is of great importance for the rural economy and the country's food supply. In order to measure the impact of cattle raising and riparian corridors on the composition and diversity of benthic macroinvertebrates in Andean microbasins, nine headwater streams were selected in the municipality of Villamaría (Caldas, Colombia), five of them with riparian corridors and four without protection. During July 2013, macroinvertebrate samples were collected using D-net and Surber nets, and each streams was characterized according to water quality, channel metrics and habitat quality score. In total 98934 individuals were collected, distributed in nine classes, 17 orders, 56 families and 92 genera. Veneroidea was the most abundant order followed by Trichoptera, Diptera, Tubificida and Ephemeroptera. Coleoptera was the richest order with 28 genera, followed by Diptera with 18, Trichoptera with 11, Ephemeroptera with 10 and Odonata with 8. Streams with riparian corridors were deeper, had higher proportion of coarse substrates and Habitat Quality Score and presented higher genus diversity than those unprotected $(\mathrm{P}<0.05)$. The genera Ferrisia, Eurygerris, Heleobia and Pisidium; total nitrogen, ammonia nitrogen and silt proportion on the streambed were correlated with unprotected streams, whilst the genus Rhagovelia, the subfamily Chironominae, the Habitat Quality Score and the proportion of coarse substrates were correlated with streams with riparian corridors. This information confirms that riparian corridors help reducing the negative impact generated by cattle ranching practices and improve the provision of environmental services, and therefore it is recommended to maintain or restore the strip of riparian vegetation to protect the streams in cattle ranching landscapes. Rev. Biol. Trop. 66(3): 1244-1257. Epub 2018 September 01 .
\end{abstract}

Key words: microbasins; riparian corridors; benthos; water quality.

En Colombia la actividad ganadera es de gran importancia para la economía rural y la oferta alimentaria del país (FEDEGAN, 2006). No obstante, ejerce una significativa presión sobre la biodiversidad, debido a los impactos directos que genera sobre los ecosistemas, especialmente en bosques de trópico bajo, bosques andinos, páramos y humedales (Chará, Murgueitio, Zuluaga, \& Giraldo, 2011). Actualmente la ganadería en el país ocupa 38 millones de hectáreas del total del territorio nacional, buena parte de las cuales presenta algún nivel de degradación (Chará, Giraldo, Zúñiga, Chará-Serna, \& Pedraza, 2011). En gran medida, esto se debe a que la decisión sobre el tipo de actividad a implementar en los terrenos no tiene en cuenta el impacto a largo plazo sobre el ambiente (Chara \& Giraldo, 2011).

La contaminación, el deterioro y la pérdida de biodiversidad de organismos acuáticos, son algunas de las consecuencias negativas de la producción ganadera tradicional en la zona andina colombiana. Esto se da porque los productores eliminan parcial o totalmente 
los bosques en la zona de captación de las microcuencas y los bosques ribereños (Chará, Pedraza, Giraldo, \& Hincapié, 2007; Hansen \& Turner, 2004). Cuando se pierde el bosque protector, se genera una serie de impactos en la microcuencas, entre los que se encuentran la compactación y el deterioro acelerado de suelos, la disminución de la calidad de agua, la pérdida de hábitat y de biodiversidad (Conservación Internacional Colombia, 2007; Hernández, Erazo, Ríos, \& Benjumea, 2004). Además, desde las áreas de pastoreo fluyen agroquími$\cos$, nutrientes, materia orgánica y sedimentos hacia las corrientes de agua (Gamboa, Reyes, \& Arrivillaga 2008). Esto modifica las características físicas naturales de los sistemas lóticos, genera pérdida de hábitats y deterioro de la calidad de agua lo que a su vez afecta a los organismos acuáticos (Mancilla, Valdovinos, Azocar, Jorquera, \& Figueroa, 2009b).

Una de las comunidades acuáticas que se caracteriza por la sensibilidad a los cambios en su ambiente son los macroinvertebrados, que incluyen diversos grupos como moluscos, lombrices, sanguijuelas, platelmintos, crustáceos, ácaros y fundamentalmente los estados juveniles de varios órdenes de insectos (Reece \& Richardson, 1999). Los macroinvertebrados han sido ampliamente utilizados como indicadores del estado de conservación del ambiente donde se encuentran, debido a que algunos grupos son tolerantes mientras otros son sensibles a la alteración de las condiciones del hábitat (Alba-Tercedor, 1996). Los macroinvertebrados también son considerados uno de los componentes más importantes de los ecosistemas dulceacuícolas por su abundancia, diversidad y el rol ecológico que cumplen en la descomposición y recirculación de nutrientes y su contribución a la red trófica como alimento para otros organismos (Merritt, Dadd, \& Walker, 1992).

En la mayoría de los casos, en microcuencas de cabecera, los macroinvertebrados acuáticos dependen de los recursos que ofrece la vegetación de la ribera para su refugio y alimentación, la cual consiste principalmente en materia orgánica particulada en forma de fragmentos de plantas, algas, bacterias y detritus (Guevara, Jara, Mercado, \& Elliott, 2006; Mancilla, Valdovinos, Azócar, Henríquez, \& Figueroa, 2009a; Meza-S., Rubio-M., G-Dias, \& M-Walteros, 2012; Meza \& Rubio, 2010). En microcuencas que son afectadas por actividades agropecuarias como la ganadería, las comunidades de macroinvertebrados modifican su estructura y diversidad, producto de la desaparición de los bosques ribereños y la consecuente modificación del hábitat dentro de los arroyos (Mancilla et al., 2009b).

Un ejemplo de lo anterior se presenta en la zona ganadera del municipio de Villamaría (Caldas, Colombia), en donde si bien se conservan muchos relictos de bosques aledaños a las fuentes de agua, es común encontrar microcuencas totalmente desprotegidas en matrices de pasturas, con alteraciones importantes en su estado físico y biológico. Teniendo en cuenta que en esta zona se originan corrientes de agua que son usadas para diferentes propósitos locales y que dichas fuentes albergan una significativa biodiversidad, es importante profundizar en estudios acerca de los efectos de la ganadería sobre las fuentes de agua y el impacto que tiene la protección de dichas fuentes mediante corredores ribereños.

\section{MATERIALES Y MÉTODOS}

Área de estudio: El presente estudio se llevó a cabo en quebradas de primer orden ubicadas en la zona ganadera del municipio de Villamaría (Caldas, Colombia) a una altura de $2400 \mathrm{msnm}$, entre las coordenadas $05^{\circ} 00^{\prime} 9.7^{\prime \prime} \mathrm{N}-75^{\circ} 26^{\prime} 17.4^{\prime \prime} \mathrm{W} \& 05^{\circ} 01^{\prime} 3.7^{\prime \prime} \mathrm{N}$ - 75'27'13.6" W. Las fuentes de agua hacen parte de la cuenca del río Chinchiná, que a su vez tributa sus aguas al río Cauca.

Los sistemas ganaderos de dicha zona son de explotación intensiva a semi-intensiva con ganado de leche y de lidia en pastoreo. El pasto predominante es Kikuyo (Cenchrus clandesti$n u s$ ), aunque es frecuente la siembra de pasto Orchoro (Dactylis glomerata) y la Falsa poa (Holcus Lannatus). El manejo de las pasturas consiste principalmente en la realización de rotación de los bovinos en los potreros y el uso 
de insumos químicos para fertilización y control de plagas del pasto.

Métodos: Se seleccionaron nueve quebradas de primer orden, cinco de las cuales presentaban bosques ribereños en buen estado de conservación con un ancho de 8 a 20 metros, y cuatro quebradas que se encontraban totalmente desprotegidas con influencia directa de ganadería (sin vegetación leñosa ribereña y entrada frecuente del ganado a beber agua). Para la selección de los sitios se tuvo en cuenta que el área de captación de las microcuencas estuviera dominada por matrices de pasturas y que los arroyos no recibieran contaminación puntual por factores diferentes a los generados por la ganadería.

Se realizó una campaña de muestreo entre el 16 y 21 de julio 2013. En cada quebrada se seleccionó un tramo de $100 \mathrm{~m}$ de largo en el que se recolectaron cinco muestras de macroinvertebrados bentónicos distribuidas en los diferentes sustratos a lo largo del transecto usando una red Surber de $0.1 \mathrm{~m}^{2}$ y $500 \mu \mathrm{m}$ de apertura de malla. Adicionalmente, se tomaron muestras con una red $\mathrm{D}$ en 20 puntos distribuidos en los hábitats diferentes (piedras, charcas, vegetación acuática, hojarasca) de acuerdo a la metodología propuesta por Chará, 2004. El material se organizó en viales rotulados, se fijaron en alcohol al $96 \%$ se identificaron hasta nivel de género y/o morfo tipo con ayuda de estéreo microscopio y el uso de claves taxonómicas (Domínguez \& Fernández, 2009; Gaviria \& Gaviria Melo, 2013; Machado Cartagena \& Rincón, 1989; Muñoz De Hoyos \& Miranda, 1995; Posada-García, 2003; \& Roldán, 2003; Roldán, 1988; Springer, 2010).

Por otro lado, en tres puntos del transecto $(0,50$ y $100 \mathrm{~m})$ se evaluaron las siguientes características abióticas del cauce: profundidad promedio, ancho del cauce, ancho del banco y se estimaron visualmente las proporciones de tipos de sustrato y de patrones de la corriente. Además, se tomaron muestras puntuales de agua para realizar análisis de parámetros fisicoquímicos y bacteriológicos, los cuales incluyeron: temperatura, conductividad, oxígeno disuelto, $\mathrm{pH}$, demanda bioquímica de oxígeno (DBO), nitrógeno amoniacal, nitritos, nitratos, fósforo, coliformes fecales y totales. Las muestras fueron enviadas al Laboratorio de Análisis de Aguas y Alimentos de la Universidad Tecnológica de Pereira. Otros parámetros como oxígeno disuelto, temperatura y conductividad fueron medidos en campo utilizando un equipo multiparámetro especializado YSI 556.

Análisis de la información: Como medida de completitud del inventario se utilizó la cobertura de muestreo propuesto por Chao y Shen (2003). Para estimar la diversidad de los géneros de macroinvertebrados en quebradas con cobertura de bosque y sin cobertura, se calculan los valores de números efectivos con tres valores de q $(0,1$ y 2$)$, que son las unidades de medición de la diversidad verdadera o serie de Hill (Jost, 2006) y se compararon estadísticamente por medio de intervalos de confianza obtenidos mediante estimadores. Para la diversidad de orden 0 riqueza de especies se utilizó el estimador no paramétrico ACE (Chao \& Lee, 1992); para la diversidad de orden 1 (exponencial del índice de Shannon), se utilizó un estimador del índice de Shannon, propuesto por Chao y Shen (2003), y para casos donde no se tiene conocimiento completo de la comunidad y para la diversidad de orden 2 (inverso de Simpson) se utilizó el estimador MLE (Magurran, 1988). Todos estos estimadores fueron calculados mediante el programa SPADE (Chao, Chiu, \& Jost, 2010).

Además se determinó la diversidad alfa, beta y gama, de la zona ganadera de Villamaría con el método propuesto por Jost (2010) y se comparó la composición de macroinvertebrados en las nueve quebradas de muestreo mediante un análisis MDS, para el que se utilizó el índice de similitud de Bray Curtis. Adicionalmente se calculó el índice biótico BMWP (Biological Monitoring Working Party) modificado por Zúñiga y Cardona (2009) y un índice de riqueza total y abundancia relativa de EPT (Ephemeroptera, Plecoptera y Trichoptera), según lo propuesto por Carrera y Fierro (2001). 
Por otra parte, se determinó el índice de calidad del hábitat propuesto por Barbour, Gerritsen, Snyder y Stribling (1998) y adaptado por Chará (2004), que consiste en otorgar una puntuación de 0 a 20, donde el máximo puntaje significa la condición óptima a las siguientes características encontradas en el tramo muestreado: sustrato disponible para la colonización, colmatación espacios-piedras, caracterización de sustratos, combinación velocidad y profundidad, diversidad de piscinas, deposición de sedimentos, alteración del cauce, frecuencia de áreas con turbulencia, sinuosidad del cauce, estabilidad de las orillas y protección vegetal.

Para determinar las diferencias estadísticas de características como profundidad, ancho húmedo y ancho de banco, entre quebradas con y sin bosques ribereños se realizó una prueba t. Por otro lado, se realizó un análisis de componentes principales para obtener las variables con mayor varianza en los sitios estudiados y se aplicó un análisis de correspondencia para establecer la relación de dichas variables con el estado de protección ribereña. Los análisis fueron realizados por el programa Statgraphics Centurion XVI (2010).

\section{RESULTADOS}

Macroinvertebrados: En total se recolectaron 98934 individuos, distribuidos en nueve clases, 17 órdenes, 56 familias y 92 géneros. Veneroida fue el orden que presentó mayor número de individuos, seguido de Diptera, Trichoptera, Tubificida y Ephemeroptera. Coleoptera fue el orden que presentó mayor riqueza de géneros con un total de 28, seguido de Diptera con 18, Trichoptera con 11 y Ephemeroptera con 10. En el total de quebradas evaluadas, la riqueza fue de 92 géneros, cuya diversidad es igual a la que tendría una comunidad virtual con 19.16 géneros en la que todas las especies tendrían las mismas abundancias (Cuadro 1). La cobertura de muestreo fue del $100 \%$ tanto para quebradas con bosques ribereños como para aquellas sin protección.

La mayor abundancia total de individuos se obtuvo en el grupo de quebradas sin bosques ribereños (68 882 individuos). Dentro de estos, Pisidium (Bivalvia) presentó el mayor número de organismos (52278), seguido de la subfamilia Naidinae género Nai (9283) y Dugesia (1 886). En las quebradas con bosque ribereño, la abundancia total fue de 30052 individuos, y el género que presentó mayor abundancia fue Simulium con 9178, seguido de Helicopsyche con 8983 y Baetodes con 5257 (Fig. 1). Se obtuvieron 23 taxones exclusivos de las quebradas con bosques ribereños y 21 de las quebradas sin protección. Coleoptera y Diptera fueron los órdenes que presentaron mayor número de géneros exclusivos en las quebradas con bosques ribereños (siete géneros cada uno), seguidos de Ephemeroptera con tres. En las quebradas sin bosque ribereño Coleoptera presentó seis géneros exclusivos, seguido por Basommatophora con tres (Cuadro 2).

La riqueza de géneros fue similar para los dos grupos de quebradas evaluadas: con bosque ribereño (71) y sin bosque ribereño (70), lo cual no permitió encontrar diferencias significativas entre los sitios. Sin embargo, con la diversidad orden uno $\left({ }^{1} \mathrm{D}\right) \mathrm{y}$ orden dos $\left({ }^{2} \mathrm{D}\right)$ si se encontraron diferencias estadísticas considerando los intervalos de confianza al $95 \%$ (Fig. 2B).

En análisis de similitud de Bray Curtis para la diversidad $\left({ }^{1} \mathrm{D}\right)$, las nueve microcuencas forman tres ensambles con una similitud del $20 \%$ (Fig. 3). Dentro de estos, aparecen dos grupos principales (similitud del $40 \%$ ), el primero está conformado por las quebradas desprotegidas, a excepción de La Edelmira, que

\section{CUADRO 1}

Diversidad alfa, beta y gama de la comunidad de macroinvertebrados en nueve arroyos con influencia ganadera en Villamaría, Caldas, Colombia

\section{TABLE 1}

Alpha, beta and gamma diversity of the macroinvertebrate community in nine streams influenced by cattle raising in Villamaría, Caldas, Colombia

\begin{tabular}{lccc} 
& ${ }^{0} \mathrm{D}$ & ${ }^{1} \mathrm{D}$ & ${ }^{2} \mathrm{D}$ \\
Alpha & 39 & 6.2 & 2.93 \\
Gamma & 92 & 19.16 & 11.74 \\
Beta & 2.36 & 3.09 & 4.01 \\
\hline
\end{tabular}




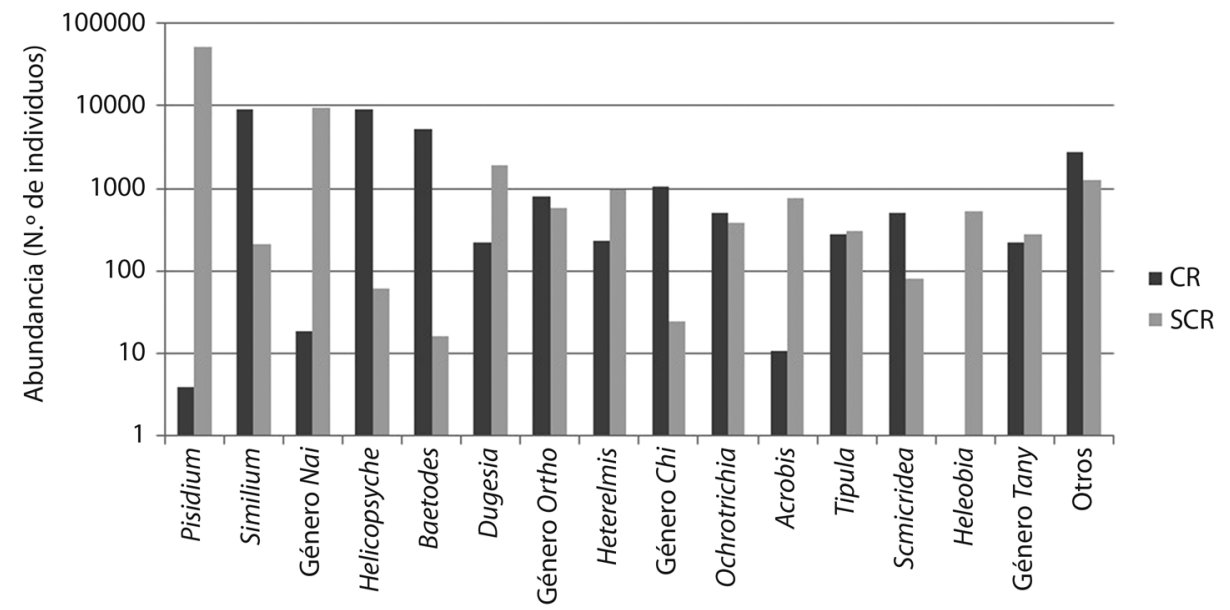

Fig. 1. Abundancia absoluta, en escala logaritmica, de géneros de macroinvertebrados presentes en nueve arroyos con corredor ribereño (CR) y sin corredor ribereño (SCR) en un paisaje ganadero de Villamaría, Caldas, Colombia.

Fig. 1. Absolute abundances, on a logarithmic scale, of macroinvertebrate genera present in the nine streams with riparian corridors (CR) and without forests (SCR) studied in a cattle raising landscape of Villamaría, Caldas, Colombia.
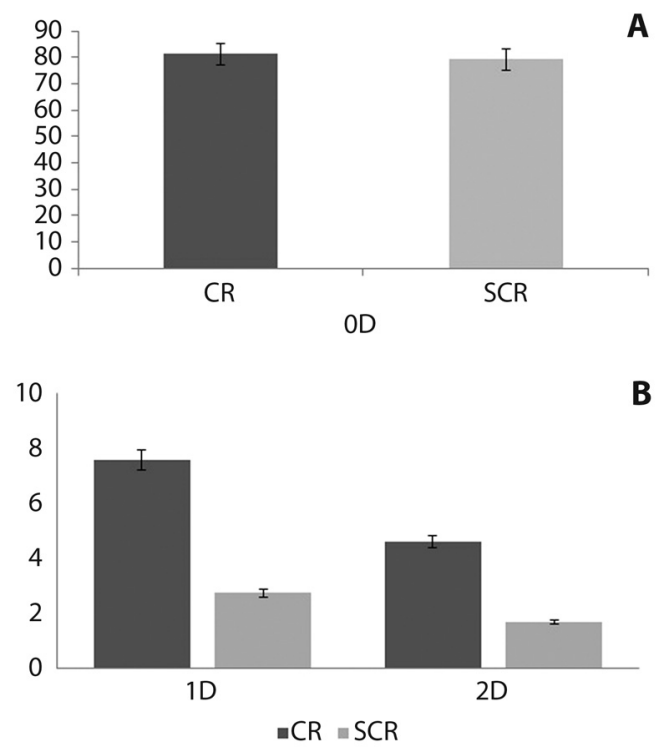

Fig. 2. Diversidad estimada de (A) $\left({ }^{0} \mathrm{D}\right)$, (B) $\left({ }^{1} \mathrm{D}\right)$ y $\left({ }^{2} \mathrm{D}\right)$ según orden de macroinvertebrados acuáticos en arroyos con corredores ribereños (CR) y sin bosque (SCR) en un paisaje ganadero en Villamaría, Caldas, Colombia. Las barras de error son intervalos de confianza al $95 \%$.

Fig. 2. Estimated diversity of (A) $\left({ }^{0} \mathrm{D}\right)$, (B) $\left({ }^{1} \mathrm{D}\right)$ and $\left({ }^{2} \mathrm{D}\right)$ order of aquatic macroinvertebrates in streams with riparian corridors (CR) and without forests (SCR) in a cattle raising landscape in Villamaría, Caldas, Colombia. Error bars are confidence intervals at $95 \%$.
A se encuentra en el segundo grupo conformado por la mayoría de microcuencas con bosque ribereño. Un tercer ensamble está compuesto por la comunidad de macroinvertebrados de la quebrada Los Arboles (con bosque ribereño), el cual tiene una similitud aproximada del $36 \%$ con las demás quebradas que están en el grupo de las protegidas por vegetación ribereña.

De acuerdo con el índice BMWP los dos grupos de quebradas obtuvieron una calificación de aguas muy limpias, ya que la puntuación obtenida fue mayor a 120, mientras que con el EPT (Ephemeroptera, Plecoptera y Trichoptera), las quebradas sin bosque ribereños presentaron una calificación deficiente $(<24)$ y las que poseen cobertura de bosque una calificación de calidad buena $(>50)$.

Características biofísicas: Las fuentes de agua con bosque ribereño son significativamente más profundas $(\mathrm{P}=0.0009)$ y tienden a ser más anchas que los arroyos desprotegidos. Además, los ambientes acuáticos con bosques ribereños presentaron mayor porcentaje de grava $(\mathrm{P}=0.044)$, y significativamente menor porcentaje de limo $(\mathrm{P}=0.019)$ (Fig. 4A, Cuadro 5). Por otro lado, el índice de calidad de 
CUADRO 2

Géneros exclusivos de macroinvertebrados en arroyos con bosques ribereños (CR) y sin bosques (SCR) en un paisaje ganadero de Villamaría, Caldas, Colombia

TABLE 2

Exclusive genera of macroinvertebrates in streams with forests in the riparian zone (CR) and without forests (SCR) in a cattle raising landscape of the Villamaría, Caldas, Colombia

\begin{tabular}{|c|c|c|c|c|}
\hline Orden & Familia & Subfamilia/Género & CR & SCR \\
\hline Tubificada & Naidinae & Tubificinae & 5 & \\
\hline \multirow[t]{3}{*}{ Basommatophora } & Ancylidae & Ferrisia & & 37 \\
\hline & Hydrobiidae & Heleobia & & 531 \\
\hline & Lymnaeidae & Lymnaea & & 60 \\
\hline Gnathobdellae & Archychobdellidae & Género C & & 7 \\
\hline \multirow[t]{13}{*}{ Coleoptera } & Curculionidae & Género F & 1 & \\
\hline & Dysticidae & Género G & & 1 \\
\hline & & Lancetes & 2 & \\
\hline & Elmidae & Austrolimnius & & 2 \\
\hline & & Género H & & 19 \\
\hline & & Género I & 2 & \\
\hline & & Elminae 1 & 5 & \\
\hline & & Neocylloepus & & 1 \\
\hline & & Onychelmis & & 25 \\
\hline & & Phanocerus & 4 & \\
\hline & Hydraenidae & Hydraena & & 1 \\
\hline & Hydrophilidae & Berosus & 2 & \\
\hline & Lampyridae & Género K & 1 & \\
\hline \multirow[t]{9}{*}{ Diptera } & Blepharoceriidae & Género M & 333 & \\
\hline & Ceratopogonidae & Maruina & & 1 \\
\hline & Empididae & Chelifera & 1 & \\
\hline & Muscidae & Limnophora & 29 & \\
\hline & Psychodidae & Género O & 10 & \\
\hline & & Maruina & 8 & \\
\hline & Tipulidae & Hexatoma & 4 & \\
\hline & & Limonia & & 6 \\
\hline & & Molophilus & 104 & \\
\hline \multirow[t]{3}{*}{ Ephemeroptera } & & Camelobaetidius & 34 & \\
\hline & Leptophlebiidae & Farrodes & 24 & \\
\hline & & Thraulodes & 1 & \\
\hline \multirow[t]{3}{*}{ Hemiptera } & Gerridae & Eurygerris & 12 & \\
\hline & Mesoveliidae & Mesovelia & & 63 \\
\hline & Veliidae & Rhagovelia & & 3 \\
\hline \multirow[t]{2}{*}{ Lepidoptera } & Cosmopterigidae & Lymnaecia & & 3 \\
\hline & Crambidae & Género Q & & 12 \\
\hline \multirow[t]{2}{*}{ Megaloptera } & Corydalidae & Corydalus & & 1 \\
\hline & Aeshnidae & Género R & & 119 \\
\hline \multirow[t]{4}{*}{ Odonata } & Aeshnidae & Aeshna & 4 & \\
\hline & Coenagrionidae & Argia & & 12 \\
\hline & Libelluidae & Género T & & 6 \\
\hline & Libelluidae & Dythemis & 1 & \\
\hline \multirow[t]{4}{*}{ Trichoptera } & Calamoceratidae & Banyallarga & 158 & \\
\hline & Leptoceridae & Triplectides & 1 & \\
\hline & & Oxyethira & & 14 \\
\hline & & & 746 & 924 \\
\hline
\end{tabular}


CUADRO 3

Índices bióticos de calidad de agua (\% EPT y BMWP) e índice de calidad de hábitat (ICH), para arroyos con corredor ribereño (CR) y sin corredor ribereño (SCR) en un paisaje ganadero de Villamaría, Caldas, Colombia

\section{TABLE 3}

Water quality biotic indexes (\% EPT and BMWP) and habitat quality index $(\mathrm{ICH})$, for streams with riparian forest (CR) and unprotected streams (SCR) in a cattle raising landscape of Villamaría, Caldas, Colombia

\begin{tabular}{lccc}
\multicolumn{1}{c}{ Índices } & $\begin{array}{c}\mathrm{CR} \\
(\mathrm{n}=5)\end{array}$ & $\begin{array}{c}\mathrm{SCR} \\
(\mathrm{n}=4)\end{array}$ & Valor-P \\
Calidad de hábitat (ICH) & 138 & 47.75 & 0.019 \\
EPT \% & 54.56 & 0.87 & 0.45 \\
BMWP-Univalle & 235 & 197 & 0.67 \\
\hline
\end{tabular}

hábitat ( $\mathrm{ICH})$ fue significativamente mayor en los arroyos con bosques ribereños $(\mathrm{P}=0.019)$ (Cuadro 1, Cuadro 5).

Parámetros fisicoquímicos y bacteriológicos: El cuadro 5 resume los parámetros evaluados en los diferentes sitios de muestreo. Aunque no se encontraron diferencias estadísticas significativas, las fuentes de agua sin
CUADRO 4

Variables con diferencias significativas entre arroyos con corredor ribereño (CR) y quebradas desprotegidas (SCR) en un paisaje ganadero de Villamaría, Caldas, Colombia

TABLE 4

Variables with significant differences between streams with forests in the riparian zone (CR) and without forests (SCR) in a cattle landscape of Villamaría,

\section{Caldas, Colombia}

\begin{tabular}{lccc}
\multicolumn{1}{r}{ Variables } & Valores CR & Valores SCR & Valor-P \\
Acrobis & 71 & 761 & 0.020 \\
Eurygerris & 12 & & 0.042 \\
Ferrisia & & 37 & 0.042 \\
Heleobia & & & 0.011 \\
Género Chi & 1065 & 24 & 0.018 \\
Pisidium & 4 & 52278 & 0.015 \\
Rhagovelia & & 3 & 0.045 \\
ICH & 138 & 47.75 & 0.019 \\
Grava & 27 & 5 & 0.044 \\
Limo & 14 & 77.5 & 0.019 \\
\hline
\end{tabular}

Prueba W de Mann-Whitney (Wilcoxon) para comparar medianas.

Mann-Whitney W test (Wilcoxon) to compare medians.

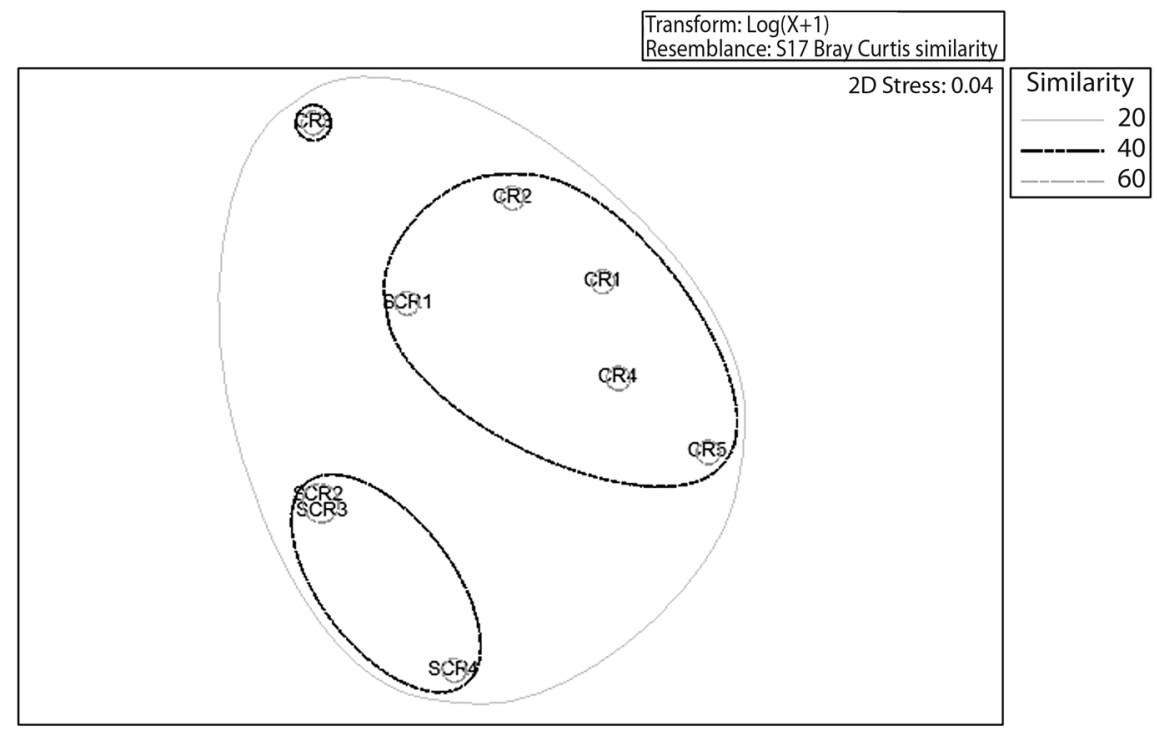

Fig. 3. Análisis de similitud de Bray Curtis, MDS, de la comunidad de macroinvertebrados en nueve arroyos ubicados en la zona ganadera de Villamaría, Caldas, Colombia.

Fig. 3. Bray Curtis similarity analysis, MDS, of the macroinvertebrate community in nine streams located in the cattle ranching area of Villamaría, Caldas, Colombia. 
CUADRO 5

Parámetros físicoquímicos y bacteriológicos en arroyos con corredor ribereño (CR) y sin corredor ribereño (SCR) en un paisaje ganadero de Villamaría, Caldas, Colombia

TABLE 5

Physicochemical and bacteriological parameters in streams with forests in the riparian zone (CR) and without forests (SCR) in a cattle raising landscape of Villamaría, Caldas, Colombia

\begin{tabular}{llc|c}
\multicolumn{1}{c}{ Parámetros } & \multicolumn{1}{c|}{ Unidad } & \multicolumn{1}{c}{ CR } & \multicolumn{1}{c}{ SCR } \\
Temperatura & ${ }^{\circ} \mathrm{C}$ & 14.736 & 15.0075 \\
$\mathrm{pH}$ & Unidades & 7.786 & 7.525 \\
Oxígeno disuelto & $\mathrm{mg} / \mathrm{L}$ & 8.282 & 7.355 \\
Conductividad eléctrica & $\mu \mathrm{S} / \mathrm{cm}$ & 82.2 & 75.5 \\
Turbiedez & $\mathrm{NTU}$ & 5.532 & 6.265 \\
Alcalinidad total & $\mathrm{mg} \mathrm{CaCO} / \mathrm{L}$ & 38.8 & 45 \\
Dureza total & $\mathrm{mg} \mathrm{CaCO} / \mathrm{L}$ & 35.6 & 31.75 \\
Nitritos & $\mathrm{mg} \mathrm{NO} / \mathrm{L}$ & 0.006 & 0.010075 \\
Nitratos & $\mathrm{mg} \mathrm{NO} / \mathrm{L}$ & 0.23 & 0.23 \\
Fósforo Total & $\mathrm{mg} \mathrm{P} / \mathrm{L}$ & 0.08 & 0.0925 \\
DBO ${ }_{5-20^{\circ} \mathrm{C}}$ & $\mathrm{mg} / \mathrm{L}$ & 2.16 & 2.25 \\
Nitrógeno Amoniacal & $\mathrm{mg} \mathrm{N}-\mathrm{NH} 3 / \mathrm{L}$ & 0.432 & 0.6725 \\
Nitrógeno Total & $\mathrm{mg} \mathrm{N} / \mathrm{L}$ & 0.91 & 1.5 \\
Sólidos Suspendidos Totales & $\mathrm{mg} / \mathrm{L}$ & 9.404 & 15.5 \\
Sólidos Sedimentables & $\mathrm{mg} / \mathrm{L}$ & 0.1 & 0.15 \\
Coliformes Totales & $\mathrm{UFC} / 100 \mathrm{ml}$ & 1100 & 1125 \\
Escherichia coli $_{\text {Profundidad }}$ & $\mathrm{UFC} / 100 \mathrm{ml}$ & 514 & 650 \\
\hline & $\mathrm{cm}$ & 5.91 & 9.11 \\
\hline
\end{tabular}

bosques ribereños presentaron menores valores en el oxígeno disuelto y mayores de turbidez, nitrógeno total, sólidos suspendidos totales y $E$. Coli, que los sitios con bosques ribereños.

Relación entre variables: Al realizar el análisis de componentes principales con todas las variables evaluadas en las quebradas (biológicas, físicas y químicas) 13 presentaron mayor varianza y diez de ellas presentaron diferencias significativas $(\mathrm{P}<0.05)$ entre quebradas con y sin bosques ribereños (Cuadro 5). Los géneros Acrobis, Ferrisia, Eurygerris, Heleobia, Pisidium, las variables ambientales nitrógeno total, nitrógeno amoniacal y el limo como característica física del cauce, están relacionados con las quebradas desprotegidas, mientras las quebradas con bosque ribereño estuvieron relacionadas con el género Rhagovelia, la subfamilia Chironominae, el valor total del índice

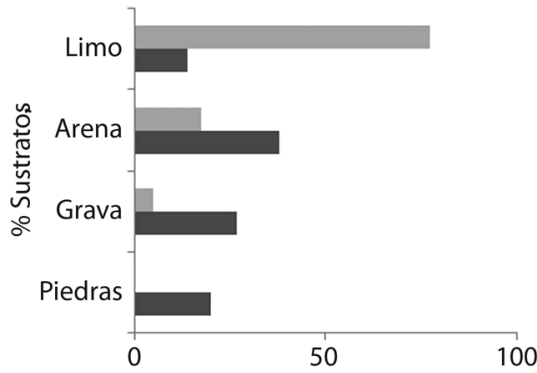

A

SCR

$\because \mathrm{CR}$

B

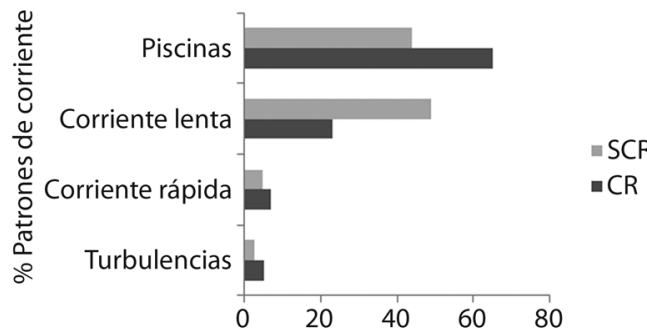

Fig. 4. Tipos de sustratos (a) y patrones de corrientes (b) en arroyos con corredor ribereño (CR) y sin corredor ribereño (SCR) estudiados un paisaje ganadero de Villamaría, Caldas, Colombia.

Fig. 4. Types of substrates (a) and current patterns (b) in streams with riparian corridors (CR) and unprotected (SCR) in a catle ranching landscape of Villamaría, Caldas, Colombia.

de calidad de hábitat, y la proporción de grava y piedras (Fig. 5).

\section{DISCUSIÓN}

A pesar de que en general, las quebradas con similares condiciones en la región albergan una importante diversidad de fauna acuática (Ascuntar et al., 2014; Chará et al., 2011a; Chará et al., 2007; Chará-Serna, Chará, Zúñiga, Pearson, \& Boyero, 2012), en el presente estudio se encontró en gran medida que la diversidad $\left({ }^{1} \mathrm{D}\right)$ fue baja (Cuadro 1). Esto puede deberse al efecto de la aplicación continua de productos químicos en las pasturas de los predios, por escorrentía pueden llegar a las fuentes de agua y ejercer un efecto sobre las poblaciones de macroinvertebrados. No obstante, se requieren mayores estudios para corroborar esta hipótesis. 


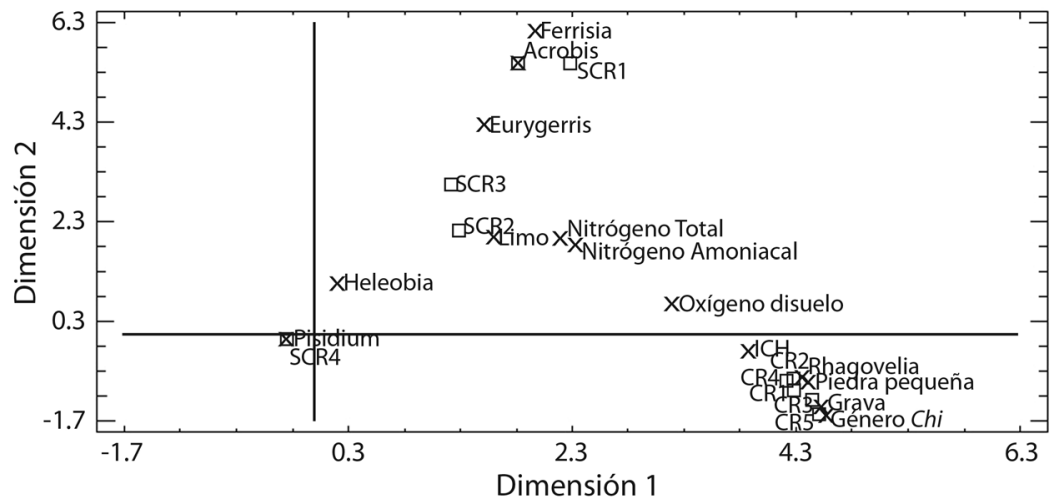

Fig. 5. Análisis de correspondencia entre las variables principales en arroyos con corredor ribereño (CR) y sin corredor ribereño (SCR) en un paisaje ganadero de Villamaría, Caldas, Colombia.

Fig. 5. Correspondence analysis between the main variables in streams with riparian corridors (CR) and without riparian corridors (SCR) in a cattle landscape of Villamaría, Caldas, Colombia.

En las quebradas evaluadas, el orden que presentó mayor riqueza de géneros fue Coleoptera. Este es uno de los grupos de organismos con más especies conocidas en la región Neotropical, con alrededor de 30 familias acuáticas o semiacuáticas asociadas a la vegetación ribereña y se encuentran en todo tipo de aguas continentales (Domínguez \& Fernández, 2009). Por tal razón, es concordante que también sea el orden que presente la mayor riqueza de géneros exclusivos en las quebradas con y sin bosques ribereños. El género más abundante para este orden en el muestreo fue Heterelmis (Elmidae), taxón muy frecuente en diferentes tipos de corrientes en Sudamérica y Colombia (Zúñiga, Chará, Giraldo, Serna, \& Pedraza, 2013).

A pesar de la baja diversidad en general, los resultados de este estudio demuestran que los bosques ribereños en zonas ganaderas tienen un efecto positivo sobre la composición de macroinvertebrados en quebradas de la zona. Esto se puede observar en los valores de los índices de diversidad exponencial de Shannon $\left({ }^{1} \mathrm{D}\right)$ y el inverso de Simpson $\left({ }^{2} \mathrm{D}\right)$ que fueron notablemente superiores en las fuentes de agua con bosques ribereños. Además, dicho grupo incluyó en su composición taxones exclusivos de los órdenes Diptera (Blephariceriidae, Limnophora, Hexatoma y Molophilus), Ephemeroptera (Camelobaetidius, Farrodes y Thraulodes), Trichoptera (Banyallarga y Triplectides) los cuales son considerados, en general, indicadores de buena calidad de agua (Carrera \& Fierro, 2001; Zúñiga \& Cardona, 2009).

Por otro lado, los géneros más abundantes en quebradas con bosques ribereños fueron Helicopsyche, Baetodes y Simulium, los cuales se caracterizan por preferir ambientes bien oxigenados, sustratos rocosos, aguas claras, aunque son tolerantes a ciertos grados de contaminación por materia orgánica (Domínguez \& Fernández, 2009; Muñoz de Hoyos \& Coscarón, 1999; Springer, 2006).

La baja diversidad y equidad de macroinvertebrados en las quebradas sin bosques ribereños se debe a que en éstas el género Pisidium (Bivalvia) fue muy dominante y el género Dugesia y género Nai de la subfamilia Naidinae presentaron un gran número de individuos. Se ha reportado que poblaciones abundantes de dichos organismos son indicadores de contaminación por materia orgánica con fuerte influencia agropecuaria (Martins, Stephan, \& Alves, 2008; Posada, Roldán, \& Ramírez, 2000; Zúñiga et al., 2013). Dentro de los taxones exclusivos y más comunes que componen las fuentes de agua sin bosques en la zona ribereña se encuentran: Heleobia, Aeshnidae, Lymnaea, Ferrisa y Mesovelia; los cuales también son considerados indicadores de contaminación por materia orgánica (Domínguez \& Fernández, 2009). 
El índice de calidad de hábitat (ICH), el análisis de Bray Curtis y el análisis de correspondencia coinciden en mostrar dos grupos principales, uno compuesto en su mayoría por las quebradas con bosques ribereños y el otro por los sitios que no presentan bosques protectores. El primer grupo se caracterizó por tener una variedad de sustratos donde predominan piedras y grava y diferentes patrones de corrientes imperando los rápidos; estas propiedades contribuyen a incrementar la diversidad de hábitats que albergan un mayor número de especies de macroinvertebrados (Chará et al., 2011a; Chará et al., 2007).

Quebradas sin presencia de bosque en su área de captación y un uso intensivo del suelo para la ganadería son factores que influyen negativamente en la diversidad y salud de los ecosistemas acuáticos, evidencia encontrada en los resultados de este estudio. Fuentes de agua sin bosques ribereños se caracterizan por presentar sustratos en los que predomina el limo, niveles altos de nitrógeno amoniacal y total, y géneros de gasterópodos como Heleobia, Acrobis y Ferrisa con abundancias significativamente altas $(\mathrm{P}<0.05)$; estos organismos pueden sobrevivir a dichas condiciones por sus hábitos alimenticios raspadores (consumo de algas y biomasa que está firmemente adherida a superficies minerales). Se consideran indicadores de eutrofización cuando se encuentran en altas cantidades (Giller \& Malmqvist, 1998; Oyaga et al., 2005).

El índice BMWP no fue muy sensible, y puede deberse a que las diferencias en las quebradas con y sin protección se dieron por la dominancia de ciertos grupos y no por la riqueza, siendo muy similar para ambos tipos de cobertura. Estas diferencias fueron mejor detectadas por el indicador de abundancia relativa de EPT, el cual tiene en cuenta no solo la presencia de los órdenes Ephemeroptera, Plecoptera y Trichoptera, sino también su abundancia con lo que puede detectar alteraciones más sutiles (Barrionuevo, Romero, Navarro, Meoni, \& Fernández, 2007; González, Ramírez, Meza, \& Dias, 2012).

\section{AGRADECIMIENTOS}

Al Departamento Administrativo de Ciencia, Tecnología e Innovación, COLCIENCIAS por la cofinanciación del proyecto "Efecto de los bosques ribereños sobre la prestación de servicios ambientales relacionados con la calidad del agua y el flujo de materia orgánica en microcuencas con influencia ganadera en el Eje Cafetero Colombiano". A Milton Montaño, Ricardo Cardozo-Zúñiga, Armando Ortega, por su colaboración en el trabajo de campo. A los propietarios de las fincas por permitir el acceso a los sitios de trabajo y su apoyo logístico, así como a los revisores anónimos que mejoraron el manuscrito con sus oportunas sugerencias.

\section{RESUMEN}

En Colombia la actividad ganadera es de gran importancia para la economía rural y la oferta alimentaria del país. Con el fin de determinar el impacto de la ganadería y de los corredores ribereños sobre la comunidad de macroinvertebrados acuáticos en microcuencas Andinas, se seleccionaron nueve fuentes de agua de cabecera en el municipio de Villamaría (Caldas, Colombia), cinco de ellas con bosques ribereños y cuatro totalmente desprotegidas. En cada quebrada durante junio 2013 se recolectaron macroinvertebrados acuáticos usando una red D y una Surber, se tomaron muestras de agua, se midieron características del cauce y se determinó el índice de calidad de hábitat. En total se recolectaron 98934 individuos distribuidos en nueve clases, 17 órdenes, 56 familias y 92 géneros. Veneroida fue el orden que presentó mayor abundancia seguido de Trichoptera, Diptera, Tubificida y Ephemeroptera. Coleoptera fue el orden que presentó mayor riqueza de géneros con 28, seguido de Diptera con 18, Trichoptera con 11, Ephemeroptera con 10 y Odonata con ocho. Las quebradas con corredor ribereño fueron significativamente más profundas, tuvieron mayor proporción de grava e Índice de Calidad de Hábitat y presentaron mayor riqueza de géneros que las quebradas desprotegidas $(\mathrm{P}<0.05)$. Los géneros Acrobis, Ferrisia, Eurygerris, Heleobia y Pisidium, el nitrógeno total, el nitrógeno amoniacal y la proporción de limo, estuvieron correlacionados con las quebradas sin bosques ribereños, mientras que el género Rhagovelia, la subfamilia Chironominae, el índice de calidad de hábitat y la proporción de grava y piedras se correlacionaron con arroyos que tienen bosques ribereños. Los resultados de este estudio confirman que la presencia de bosques en zonas de ribera ayuda a amortiguar el impacto negativo que generan las prácticas ganaderas sobre las microcuencas y mejoran los servicios ambientales que estas prestan, por lo que se recomienda mantener la franja 
de vegetación ribereña en arroyos que la poseen y permitir el establecimiento de esta en arroyos desprovistos de bosque en sus las laderas.

Palabras clave: microcuencas; bentos; calidad de agua; bosques ribereños.

\section{REFERENCIAS}

Alba-Tercedor, J. (1996). Macroinvertebrados acuáticos y calidad de las aguas de los ríos. Simposio del Agua en Andalucia, II, 203-213.

Ascuntar, O., Zuñiga, M. del C., Giraldo, L., Ramírez, Y., Chará, J., \& Ramos, B. (2014). Contribución al conocimiento de los tricópteros adultos (Insecta: Trichoptera) de los andes orientales de colombia. Cali: CIPAV.

Barbour, M. T., Gerritsen, J., Snyder, B. D., \& Stribling, J. B. (1998). Rapid bioassessment protocols for use in streams and wadeable rivers: periphyton, benthic macroinvertebrates and fish, Second Edition. U.S Environmental Protection Agency; Office of Water: Washington, D.C. DOI: EPA 841-B-99-002

Barrionuevo, M. A., Romero, F., Navarro, M. G., Meoni, G. S., \& Fernández, H. (2007). Monitoreo de la calidad del agua en un río subtropical de montaña: El río Lules (Tucummán, Argentina). Conagua

Carrera, C., \& Fierro, K. (2001). Manual de monitoreo: Los macroinvertebrados acuáticos como indicadores de la calidad del agua. O. Z. Mendoza (Ed.). Quito: EcoCiencia.

Chao, A., Chiu, C. H., \& Jost, L. (2010). Phylogenetic diversity measures based on Hill numbers. Philosophical Transactions of the Royal Society of London. Series B, Biological Sciences, 365, 3599-3609. DOI: 10.1098/rstb.2010.0272

Chao, A., \& Lee, S. (1992). Estimating the number of classes via sample coverage. Journal of the American Statistical Association, 87, 210-217. DOI: $10.1080 / 01621459.1992 .10475194$

Chao, A., \& Shen, T. J. (2003). Nonparametric estimation of Shannon's index of diversity when there are unseen species in sample. Environmental and Ecological Statistics, 10, 429-443. DOI: 10.1023/A:1026096204727

Chará, J. D. (2004). Manual para la evaluación biológica de ambientes acuáticos en microcuencas ganaderas. J. D. Chará (Ed.). Cali: Fundación Centro para la Investigación en Sistemas Sostenibles de Producción Agropecuaria. CIPAV.

Chara, J. D., \& Giraldo, C. (2011). Servicios ambientales de la biodiversidad en paisajes agropecuarios. Cali: Fundación CIPAV.
Chará, J. D., Giraldo, L. P., Zúñiga, M. del C., Chará-Serna, A. M., \& Pedraza, G. (2011). Cambios en el ambiente acuático asociados a la restauración del corredor ribereño en una quebrada afectada por ganadería en la cuenca del río la vieja, Colombia. En I Congreso Colombiano de restauración ecológica \& II simposio nacional de experiencias en restauración ecológica. DOI: 978-958-719-741-9

Chará, J. D., Murgueitio, E., Zuluaga, A., \& Giraldo, C. (Eds.). (2011). Ganadería colombiana sostenible. Cali: Fundación CIPAV.

Chará, J. D., Pedraza, G., Giraldo, L. P., \& Hincapié, D. (2007). Efecto de los corredores ribereños sobre el estado de quebradas en la zona ganadera del río La Vieja, Colombia. Agroforestería en las Américas, $45,72-78$.

Chará-Serna, A., Chará, J., Zúñiga, M., Pearson, R., \& Boyero, L. (2012). Diets of leaf litter-associated invertebrates in three tropical streams. Annales de Limnologie - International Journal of Limnology, 48, 139-144. DOI: 10.1051/limn/2012013

Conservación Internacional Colombia. (2007). Plan de Manejo de los páramos del departamento de Caldas. Manizales. Recuperado de http://www.corpocaldas. gov.co/publicaciones/639/Plan_de_Manejo_de_Paramos-Caldas.pdf

Domínguez, E., \& Fernández, H. (Eds.). (2009). Macroinvertebrados bentónicos sudamericanos, Sistemática y Biología. San Miguel Tucumán: Fundación Miguel Lillo.

FEDEGAN. Federación Colombiana de Ganaderos. (2006). Plan estratégico de la ganadería 2019. FEDEGAN (Ed.). Bogotá D.C.

Gamboa, M., Reyes, R., \& Arrivillaga, J. (2008). Revisiones Macroinvertebrados bentónicos como bioindicadores de salud ambiental. Boletín De Malariología y Salud Ambiental, XLVIII(2002), 109-120.

Gaviria, E., \& Gaviria Melo, S. (2013). Guía de laboratorio sobre los Oligochaeta. Tunja: Universidad Pedagógica y Tecnológica de Colombia, UPTC.

Giller, P. S., \& Malmqvist, B. (1998). The biology of streams and rivers. Biology of habitats. Recuperado de http://books.google.com/books?hl=en\& amp;1r=\&amp;id=FZLVrauq0ZMC\&amp;oi $=$ fnd $\& a m p ; p g=$ PA $1 \& a m p ; d q=$ The + Biology + of + Strea $\mathrm{ms}+$ and + Rivers\&amp;ots $=$ JomxRGTNiz\&amp;s ig=_adM3FUw6QqDreQgB5MTJPzTi3w

González, S. M., Ramírez, Y. P., Meza, A. M., \& Dias, L. (2012). Diversidad de macroinvertebrados acuáticos y calidad de agua de quebradas abastecedoras del municipio de Manizales. Boletín Científico Museo de Historia Natural, 16(2), 135-148. 
Guevara, G., Jara, C., Mercado, M., \& Elliott, S. (2006). Comparación del macrozoobentos presente en arroyos con diferente tipo de vegetación ribereña en la Reserva Costera Valdiviana, Sur de Chile. Asociación Colombiana de Limnología, 1(November 2005), 98-105.

Hansen, A. J., \& Turner, W. (2004). Land Use Change and Biodiversity: A Synthesis of Rates and Consequences during the Period of Satellite Imagery. Land Use Change and Biodiversity, 6, 277-299. DOI: 10.1007/978-1-4020-2562-4_16

Hernández, S., Erazo, J., Ríos, C. I., \& Benjumea, F. (2004). Diseño de instrumentos de política para la conservación y uso sostenible de la biodiversidad en sistemas productivos en la zona alta de la cuenca del rio Chinchiná, Manizales (Caldas). Manizales.

Jost, L. (2006). Entropy and diversity. Oikos, 113, 363-375. DOI: $10.1111 /$ j.2006.0030-1299.14714.x

Jost, L. (2010). The relation between evenness and diversity. Diversity, 2, 207-232. DOI: 10.3390/d2020207

Machado Cartagena, T. A., \& Rincón, J. (1989). Distribución ecológica e identificación de los coleópteros acuáticos en diferentes pisos altitudinales del departamento de Antioquia. Universidad de Antioquia.

Magurran A. E. (1988) Diversity indices and species abundance models. In: Ecological Diversity and Its Measurement. Dordrecht: Springer. DOI: 10.1007/978-94-015-7358-0_2

Mancilla, G., Valdovinos, C., Azócar, M., Henríquez, M., \& Figueroa, R. (2009). Aproximación multimétrica a la evaluación de la calidad del agua en cuencas con diferentes niveles de intervención antrópica. Interciencia, 34, 857-864.

Mancilla, G., Valdovinos, C., Azocar, M., Jorquera, P., \& Figueroa, R. (2009). Efecto del reemplazo de la vegetación nativa de ribera sobre la comunidad de macroinvertebrados bentónicos en arroyos de climas templados, Chile central. Hidrobiologica, 19(3), 193-203.

Martins, R., Stephan, N., \& Alves, R. (2008). Tubificidae (Annelida: Oligochaeta) as an indicator of water quality in an urban stream in southeast Brazil. Acta Limnologica Brasiliensia, 20, 221-226. Recuperado de http://www.ablimno.org.br/acta/pdf/acta20_vol3_05. pdf

Merritt, R. W., Dadd, R. H., \& Walker, E. D. (1992). Feeding behavior, natural food, and nutritional relationships of larval mosquitoes. Annual Review of Entomology, 37(128), 349-376. DOI: 10.1146/annurev.en.37.010192.002025

Meza S. A. M., \& Rubio M. J. (2010). Composición y estructura trófica de macroinvertebrados acuáticos en la subcuenca alta del río chinchiná (Tesis) Universidad de Caldas, Colombia.

Meza, A. M., Rubio, J., Dias, L., \& Walteros, J. (2012). Calidad de agua y composición de macroinvertebrados acuáticos en la subcuenca alta del río Chinchiná. Caldasia, 34(2), 443-456.

Muñoz de Hoyos, P., \& Coscarón, S. (1999). Claves para la identificación de Simúlidos (Diptera: Simulidae) presentes entre las vertientes magdalenense y orinocense, en un sector al centro de Colombia. Revista Académica Colombiana de Ciencias Exactas, Físicas y Naturales, 23, 181-214.

Muñoz de Hoyos, P., \& Miranda, D. (1995). Simulidos (diptera: simuliidae) presentes entre las vertientes magdalenense y orinocense, en un sector al centro de colombia. Caldasia, 19, 297-310.

Oyaga, R., Mosquera, D., Maury, H., Castro, J., Gallardo, L., \& Toloza, E. (2005). Bioindicadores macroinvertebrados para la evaluaciòn de contaminaciòn acuàtica, presentes en el embalse el Guajaro departamento de Atlantico. In Conferencia internacional usos múltiples del agua: Para la vida y el desarrollo sostenible (Vol. 25, pp. 44-47). Colombia: Instituto Cinara.

Posada-García, J., \& Roldán-Pérez, G. (2003). Clave ilustrada y diversidad de las larvas de Trichoptera en el Nor-Occidente. Caldasia, 25(1), 169-192.

Posada, J. A., Roldán, G. G., \& Ramírez, J. J. (2000). Caracterización físicoquímica y biológica de la calidad de aguas de la cuenca de la quebrada Piedras Blancas, Antioquia, Colombia. Revista de Biologia Tropical, 48(1), 59-70.

Reece, P. F., \& Richardson, J. S. (1999). Biomonitoring with the Reference Condition Approach for the Detection of Aquatic Ecosystems at Risk. Proceedings of a Conference on the Biology Management of Species and Habitats at Risk, 549-552.

Roldán, G. (1988). Guía para el estudio de los macroinvertebrados acuáticos del Departamento de Antióquia. En E. Pérez Arbeláez (Ed.). Medellín: Universidad de Antioquia.

Springer, M. (2006). Clave taxonómica para larvas de las familias del orden Trichoptera (Insecta) de Costa Rica. Revista de Biología Tropical, 54, 273-286. DOI: 0034-7744

Springer, M. (2010). Trichoptera. Capítulo 7. Revista de Biología Tropical, 58, 151-198.

Statgraphics Centurion. (2010). Graphics. http://info.statgraphics.com/statgraphics-home

Zúñiga, M. del C., \& Cardona, W. (2009). Bioindicadores de calidad de agua y caudal ambiental. En J. Cantera, J. Carvajal, \& L. M. Castro (Eds.), Caudal ambiental: Conceptos, experiencias y desafios (Programa editorial, pp. 167-197). Cali: Universidad del Valle.

Zúñiga, M. del C., Chará, J., Giraldo, L. P., Serna, A. M. C.,

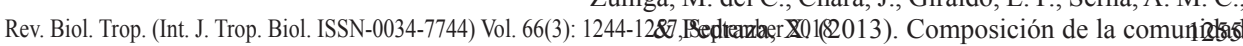
de macroinvertebrados acuáticos en pequeñas quebradas de la región andina colombiana, con énfasis en la entomofaunal. Dugesiana, 20(2), 263-277. 


\section{APÉNDICE 1}

Composición macroinvertebrados acuáticos colectados en arroyos con corredor ribereño (CR) y sin corredor ribereño (SCR) en un paisaje ganadero de Villamaría, Caldas, Colombia

\section{APÉNDICE 1}

Aquatic macroinvertebrates composition collected in streams with riparian corridor (CR) and without riverside corridor (SCR) in a cattle landscape of the municipality of Villamaría, Caldas, Colombia)

\begin{tabular}{|c|c|c|c|c|c|}
\hline Clase & Orden & Familia & Género & $\mathrm{CR}$ & SCR \\
\hline Arachnida & Acari & Lymnessiidae & Género A & 2 & 1 \\
\hline Bivalvia & Veneroida & Sphaeriidae & Pisidium & 4 & 52278 \\
\hline \multirow[t]{2}{*}{ Clitellata } & Tubificada & Naidinae & Género Tub & 5 & \\
\hline & & & Género Nai & 19 & 9283 \\
\hline Entognatha & Collembola & & Género B & 2 & 4 \\
\hline \multirow[t]{4}{*}{ Gastropoda } & Basommatophora & Ancylidae & Ferrisia & & 37 \\
\hline & & Hydrobiidae & Heleobia & & 531 \\
\hline & & Lymnaeidae & Lymnaea & & 60 \\
\hline & & Planorbiidae & Acrobis & 11 & 761 \\
\hline \multirow[t]{2}{*}{ Hirudinea } & Gnathobdellae & Archychobdellidae & Género $\mathrm{C}$ & & 7 \\
\hline & Rhynchobdellae & Glossiphoniidae & Género D & 1 & 12 \\
\hline \multirow[t]{33}{*}{ Insecta } & Coleoptera & Chrysomelidae & Género E & 7 & 2 \\
\hline & & Curculionidae & Género F & 1 & \\
\hline & & Dysticidae & Género G & & 1 \\
\hline & & & Laccophilus & 44 & 7 \\
\hline & & & Lancetes & 2 & \\
\hline & & & Rhantus & 17 & 4 \\
\hline & & Elmidae & Austrolimnius & & 2 \\
\hline & & & Cylloepus & 26 & 6 \\
\hline & & & Heterelmis & 229 & 956 \\
\hline & & & Género Elminae 1 & 5 & \\
\hline & & & Macrelmis & 201 & 5 \\
\hline & & & Género H & & 19 \\
\hline & & & Género I & 2 & \\
\hline & & & Neocylloepus & & 1 \\
\hline & & & Onychelmis & & 25 \\
\hline & & & Phanocerus & 4 & \\
\hline & & Gyrinidae & Andogyrus & 8 & 11 \\
\hline & & Hydraenidae & Hydraena & & 1 \\
\hline & & Hydrophilidae & Género J & 1 & 4 \\
\hline & & & Anacaena & 30 & 23 \\
\hline & & & Berosus & 2 & \\
\hline & & & Hydrophilus & 7 & 15 \\
\hline & & & Tropisternus & 37 & 2 \\
\hline & & Lampyridae & Género K & 1 & \\
\hline & & Ptilodactylidae & Anchytarsus & 53 & 2 \\
\hline & & Scirtidae & Elodes & 385 & 4 \\
\hline & & Staphylinidae & Género L & 10 & 11 \\
\hline & Diptera & Blepharoceriidae & Género M & 333 & \\
\hline & & Ceratopogonidae & Alluaudomyia & 1 & 5 \\
\hline & & & Maruina & & 1 \\
\hline & & Chironomidae & Género $C h i$ & 1065 & 24 \\
\hline & & & Género Orth & 794 & 580 \\
\hline & & & Género Tany & 217 & 282 \\
\hline
\end{tabular}


APÉNDICE (Continuación) / APPENDIX (Continued)

\begin{tabular}{|c|c|c|c|c|c|}
\hline Clase & Orden & Familia & Género & CR & SCR \\
\hline & & Empididae & Género N & 3 & 1 \\
\hline & & & Chelifera & 1 & \\
\hline & & Muscidae & Género $\tilde{N}$ & 2 & 1 \\
\hline & & & Limnophora & 29 & \\
\hline & & Psychodidae & Género O & 10 & \\
\hline & & & Maruina & 8 & \\
\hline & & Simulidae & Gigantodax & 64 & 10 \\
\hline & & & Simulium & 9178 & 208 \\
\hline & & Tipulidae & Hexatoma & 4 & \\
\hline & & & Limonia & & 6 \\
\hline & & & Molophilus & 104 & \\
\hline & & & Tipula & 284 & 304 \\
\hline & Ephemeroptera & Baetidae & Andesiops & 252 & 5 \\
\hline & & & Baetodes & 5257 & 16 \\
\hline & & & Camelobaetidius & 34 & \\
\hline & & & Mayobaetis & 25 & 1 \\
\hline & & Leptohyphidae & Leptohyphes & 2 & 1 \\
\hline & & Leptophlebiidae & Farrodes & 24 & \\
\hline & & & Thraulodes & 1 & \\
\hline & Hemiptera & Gerridae & Eurygerris & 12 & \\
\hline & & Mesoveliidae & Mesovelia & & 63 \\
\hline & & Veliidae & Rhagovelia & & 3 \\
\hline & Lepidoptera & Arctiidae & Género P & 177 & 13 \\
\hline & & Cosmopterigidae & Lymnaecia & & 3 \\
\hline & & Crambidae & Género Q & & 12 \\
\hline & & & Género Nym & 4 & 2 \\
\hline & Megaloptera & Corydalidae & Corydalus & & 1 \\
\hline & Odonata & Aeshnidae & Género R & & 119 \\
\hline & & & Aeshna & 4 & \\
\hline & & Calopterygidae & Calopterygidae & 2 & 47 \\
\hline & & Coenagrionidae & Argia & & 12 \\
\hline & & Gomphidae & Género S & 1 & 314 \\
\hline & & Libelluidae & Dythemis & 1 & \\
\hline & & & Marilia & 32 & 59 \\
\hline & & & Género T & & 6 \\
\hline & Tricoptera & Calamoceratidae & Banyallarga & 158 & \\
\hline & & Glossosomatidae & Mexitrichia & 249 & 1 \\
\hline & & Helicopsychidae & Helicopsyche & 8983 & 61 \\
\hline & & Hydrobiosidae & Atopsyche & 373 & 11 \\
\hline & & Hydropsychidae & Smicridea & 500 & 80 \\
\hline & & Hydroptilidae & Ochrotrichia & 510 & 377 \\
\hline & & & Oxyethira & & 14 \\
\hline & & Leptoceridae & Triplectides & 1 & \\
\hline & & Odontoceridae & Marilia & 7 & 17 \\
\hline & & Philopotamidae & Wormaldia & 7 & 5 \\
\hline & & Polycentropodidae & Polycentropus & 1 & 8 \\
\hline Malacostraca & Amphipoda & Hyalellidae & Hyalella & 3 & 248 \\
\hline Turbellaria & Tricladida & Planariidae & Dugesia & 219 & 1886 \\
\hline & & & & 30052 & 68882 \\
\hline
\end{tabular}

